or lower in the respiratory tract and then may cause bronchitis rather than a cold. ${ }^{18}$ Thus the data suggest that the Coxsackie A21 virus is transmitted mainly in "short-range" droplets of nasal secretions expelled when sneezing or blowing the nose, but further confirmation is needed. All we know at present is that there is a chance of about one in five that other members of the family of a cold-sufferer will catch a cold from him ${ }^{17}$ and a slightly higher chance than average of catching a cold if one works in a room next to a person suffering from one. ${ }^{18}$ Apparently only a few people produce enough virus to form infected droplets at all frequently, and so it is probable that only a small proportion of patients actually spread the infection.

Why seasonal factors have such a marked effect on the incidence of colds is something of a mystery. A recent epidemiological study ${ }^{19}$ has shown that there is a correlation between a drop in the temperature outdoors and the onset of colds two or three days later. There was also a slight association with humidity. Other meteorological factors had no effect at all. It seems likely, therefore, that on a chilly day when the temperature outdoors is falling, the shedding, survival, or uptake of virus or perhaps all three are more effective than on other days. However, Sir Christopher Andrewes $^{20}$ and R. E. Hope-Simpson ${ }^{21}$ believe that a widespread virus infection in a subclinical form which cannot be detected in the laboratory is made clinically evident by seasonal changes, particularly a fall in temperature. Isolation of the patient, to a greater or less extent, still seems to be the only effective method of control.

\section{Natural History of Breast Cancer}

Cancer of the breast is a notoriously malignant tumour. It spreads relentlessly through the parenchyma and surrounding fat to the overlying skin, and soon metastasizes to the axillary lymph nodes. But its lethal effects are related to the frequency of blood-borne metastases in distant sites such as the lungs, liver, and skeleton, especially the vertebral column. The duration of life in untreated cases is usually about three years, but the range varies from a few months to over thirty years. ${ }^{1}$ Some of these tumours are hormone-dependent, and a regression may follow bilateral oophorectomy and adrenalectomywith or without hypophysectomy-but the effect is only temporary. ${ }^{2} 3$ In four cases metastases regressed spontaneously after mastectomy. ${ }^{4}$ Breast-cancer cells tend to lie dormant in the tissues for long periods of time, ${ }^{5}$ and metastases may suddenly appear as long as thirty years after the successful removal of the primary tumour. ${ }^{6}$ Intercurrent illnesses and operations sometimes act as precipitating factors. It follows, therefore, that no matter how early and thorough

\footnotetext{
Haagensen, C. D., Diseases of the Breast, 1956. Saunders, Philadelphia.

2 Brit. med. F., 1961, 1, 646.

I Ibid., 1961, 1, 1666.

- Everson, T. C., and Cole, W. H., Ann. Surg., 1956, 144, 366.

s Hadfield, G., Brit. med. F., 1954, $2,607$.

- Gordon-Taylor, G., ibid., 1959, 1, 455.

Truscott, B. M., Brit. F. Cancer, 1947, 1, 129.

B Bloom, H. J. G., ibid, 1950, 4, 259.

- ibid., 1950, 4, 347.

— and Richardson, W. W., ibid., 1957, 11, 359.

$: 1-$ and Harries, E. J., Brit. med. f., 1962, 2, 213.

12 McKinnon, N. E., Lancet, 1954, 1, 251.

13 Canad. med. Ass. F., 1955, 73, 614

i4 Brit. med. F., 1962, 2, 247.

is Bloom, H. J. G., Brit. F. Cancer, 1965, 19, 228.
}

the treatment there is a danger of recurrence and metastasis so long as the patient remains alive.

Despite the variability of the course of the disease, it is possible to predict the degree of malignancy of individual tumours with reasonable accuracy. H. J. G. Bloom and his colleagues have investigated the prognosis of breast cancer, and find that the two most important factors are the extent of spread, or stage, of the tumour and its degree of differentiation, or grade..$^{8-11}$ Thus a tumour confined to the breast itself (stage I) and well differentiated (grade I) has a much better prognosis than one which has spread to the axillary lymph nodes (stages II and III) and is very poorly differentiated (grade III). In a series of untreated patients they found that $22 \%$ with grade-I and grade-II tumours survived over five years, whereas no patient with grade-III cancer was alive after 53 months. ${ }^{11}$ Age itself bears no relation to prognosis, ${ }^{9}$ but pregnancy and lactation are periods in which particularly lethal tumours occur.

It is very common for a patient treated as promptly as possible to succumb to widespread metastases within a year. The value of early treatment in reducing the mortality rate from cancer of the breast has been seriously questioned, since sometimes spread of the tumour by the blood-stream may occur so early that interference is impractical. Furthermore, the good results of treatment in stage-I cases might be due to many of these tumours being intrinsically non-lethal and nonmetastasizing. ${ }^{12}$ But even in treated stage-I cases with grade-I tumours the survival rate falls with the passage of time, and most of the patients die of breast cancer. ${ }^{11}$ So also do the great majority of untreated cases; only $5 \%$ succumb to intercurrent disease while the tumour remains confined to the breast. ${ }^{11}$ The value of treatment is confirmed by the finding that $82 \%$ of treated cases of grade-I tumours and $33 \%$ of grade-III tumours survive over five years as compared with $22 \%$ and $0 \%$, respectively, of untreated cases. ${ }^{11} 14$

Nevertheless, it is common for patients who delay over a year before seeking help to survive at least as long as those who are treated as soon as the disease is detected. ${ }^{9}$ In a recent paper Bloom has investigated this paradox, analysing the relevant factors in great detail. ${ }^{15}$ As cancer spreads with the passage of time, and the prognosis worsens as the stage advances, it follows that delay must affect the outcome of the disease. But in tumours of great intrinsic malignancy distant spread occurs so early that even the most expeditious treatment can seldom prevent death. As the most malignant tumours tend to produce the most alarming symptoms, they are likely to be diagnosed earlier than the more slowly growing types of cancer, in which early treatment does prolong life and delay is most serious. This shows how important is the intrinsic malignancy of the individual tumour when the effects of delay or a particular therapy are being assessed in relation to survival rate.

Unfortunately it is very difficult to assess the intrinsic properties of cancer in an individual patient. As regards breast cancer, grading in terms of differentiation and nuclear morphology has been shown to correlate fairly closely with the pattern of behaviour of the tumour. ${ }^{810}$ While even tumours of low-grade malignancy may metastasize at an early stage, probably these develop slowly and become active much later than do the metastases of highly malignant tumours. ${ }^{10}$ Perhaps the intrinsic malignancy of a tumour is related to factors of host resistance, and the degree of differentiation is merely an expression of this. If this is so it might be fruitful to spend more time studying the metabolic, immunological, and psychological responses of the patient herself. 